\title{
Identificación de cianobacterias de la laguna "La Mansión" en una universidad privada confesional
}

\section{Identification of cyanobacteria of the lagoon "La Mansión" in a private denominational university}

\author{
Maycol Owen Echevarria Mendoza, Maria Alejandra Roman Medrano, Ginnie Estephany Ruíz Díaz y Gina \\ Tito Tolentino \\ ${ }^{a}$ E.P. de Ingeniería Ambiental, Facultad de Ingeniería y Arquitectura, Universidad Peruana Unión
}

\begin{abstract}
Resumen
El objetivo de este artículo es identificar los géneros de cianobacterias presentes en la laguna "La mansión" y su impacto en el ecosistema local. En este contexto, las cianobacterias tienen gran influencia, puesto que son base de esta cadena y fijan nitrógeno, a su vez, liberan grandes cantidades de oxígeno al ecosistema acuático. Ésta laguna, tiene un área de $3499 \mathrm{~m}^{2}$, y amerita realizar una segmentación en 8 sectores estratégicos para poder identificarlas, además, se colocaron mallas estándar de $40 \mathrm{~cm}^{2}$, las cuales capturaron todas las partículas suspendidas en el agua, entre ellas, las cianobacterias. A través de botellas de $225 \mathrm{ml}$ fueron trasladadas al laboratorio para ser observadas en el microscopio. Los resultados más importantes fueron la detección de 6 especies de cianobacterias, siendo la principal y más abundante la anabaena, quien se encarga en mayor escala de fijar nitrógeno y producir oxígeno frente a los demás géneros, sin embargo, el crecimiento de ésta es acelerado, lo que conllevaría en el futuro a tener un problema de eutrofización. Por lo tanto, se concluye que las cianobacterias encontradas podrían incrementar su población ocasionando muerte acuática y alterando el ecosistema en caso de no controlar los inicios del proceso eutrófico.
\end{abstract}

Palabras clave: Cianobacteria, eutrofización, anabaena, cianotoxinas.

\begin{abstract}
Abstrac
The objective of this article is to identify the cyanobacteria present in the lagoon "La mansion" and its impact on the local ecosystem. In this context, cyanobacteria have great influence, that their base of this chain and fix nitrogen, to their they release large amounts of oxygen into the aquatic ecosystem. This lagoon has an area of $3499 \mathrm{~m} 2$ and America has a segmentation in 8 strategic sectors to be able to identify them, in addition, standard meshes of $40 \mathrm{~cm} 2$ were placed, which captured all suspended particles in the water, including cyanobacteria. Through bottles of $225 \mathrm{ml}$ were transferred to the laboratory for observations under a microscope. The most important results were the detection of 6 species of cyanobacteria, the main and most abundant being the anabaena, which is responsible for a larger scale to fix nitrogen and produce oxygen compared to other genera, however the growth of this is accelerated, which would lead in the future to have a problem of eutrophication. Therefore, it is concluded that the cyanobacteria found increased population causing aquatic death and altering the ecosystem in case of not controlling the beginnings of the eutrophic process.
\end{abstract}

Keywords: Cyanobacteria, eutrophication, anabaena, cyanotoxins

\section{Introducción}

La cianobacterias son organismos procariotas Gram negativas, poseen características de las bacterias y también de las algas, las cianobacterias se encuentran finamente dispersos en el agua, pero si estas aumentan 
su población producen turbidez en el agua como es el caso de la laguna materia de este estudio, pero si el lugar presta las condiciones favorables para su desarrollo, como abundancia de nutrientes, temperatura adecuada entonces se da un crecimiento exponencial que forma los blooms. Según Hidrol, (n.d.) la presencia abundante de cianobacterias genera limitaciones para el correcto funcionamiento de la cadena trófica de este ecosistema. América del sur tiene grandes cantidades de agua dulce reservadas, pero también debido a la abundancia de materia orgánica y al existir tantos microclimas hay más afloramiento de cianobacterias y según la OMS las cianobacterias se encuentran en la lista de problemas de salud emergente. Las cianobacterias contribuyen significativamente a la producción primaria acuática, ocupando un papel central en el plancton junto con micro algas eucariotas.

Sin embargo, las cianobacterias planctónicas, también son un tema de preocupación para calidad del agua. Su participación en el ecosistema es de gran importancia ya que al ser fijadores de nitrógeno en el agua y producir oxígeno permiten el desarrollo de otros tipos de vida, si el ambiente se vuelve adecuado se proliferan y aparecen cianobacterias que pueden producir cianotoxinas que en exceso terminan intoxicando el cuerpo de agua e inhibiendo la vida normal y el desarrollo de este ecosistema acuático por lo que estaría ocasionando un impacto significativo en su ecosistema local. Para Alonso et al., (2008): La eutrofización de los ambientes acuáticos, es decir el enriquecimiento de las aguas superficiales en sustancias nutritivas ( $\mathrm{N}$ y $\mathrm{P}$ ) principalmente en aguas que no tienen tanta fluidez y movimiento, esto favorece el crecimiento de poblaciones de cianobacterias. Por lo que se estaría hablando de una probabilidad de que la condición de calidad de agua y la presencia de cianobacterias en la laguna causarían un impacto negativo dependiendo al género de cianobacterias que se establece en el cuerpo de agua, por lo cual esta genera cambios en el ecosistema, y en la calidad de agua se corre el riesgo de producir eutrofización por cianobacterias filamentosas (González-piana, 2012), cuando las cianobacterias producen las cianotoxinas entonces el impacto será más significativo. Es por esto que el objetivo fue identificar los géneros de cianobacterias presentes en la laguna "La mansión” y así entender cuál podría ser el impacto en el ecosistema acuático.

\section{Las cianobacterias}

Las Cianobacterias son organismos microscópicos, bacterias Gram negativas que contienen clorofila, lo que les permite realizar fotosíntesis (Para \& Personal, 2015). Las cianobacterias se consideran como bacterias que pueden realizar fotosíntesis, ya que tienen algunas coincidencias con las algas. Además según (Ramirez, Rojo, \& Espejel, 2007), las cianobacterias son procariotas, pueden realizar fotosíntesis, su estructura es unicelular y multicelular, pueden producir toxinas que vendrían a ser cianotoxinas, las cianobacterias pueden desarrollarse en diferentes hábitats y en el agua son visibles. Existen otras diferencias y similitudes que se pueden apreciar en la Tabla 1.

Tabla 1

Diferencias entre las algas, cianobacterias y bacterias.

\begin{tabular}{|c|c|c|}
\hline Algas & Cianobacterias & Bacterias \\
\hline \multirow[t]{2}{*}{ Eucariotas } & \multicolumn{2}{|c|}{ Procariotas } \\
\hline & Fotosintéticas & No fotosintética \\
\hline No producen toxinas & $\begin{array}{c}\text { Capaces de producir toxinas y exudarlas al medio } \\
\text { circundante }\end{array}$ & Capaces de producir toxinas \\
\hline \multicolumn{2}{|c|}{ Unicelular y multicelular } & Unicelular \\
\hline \multicolumn{2}{|c|}{ Se encuentran sólo en ambientes acuáticos } & s habitas diversos \\
\hline \multicolumn{2}{|c|}{ Pueden formar colonias visibles en el agua } & $\begin{array}{c}\text { Pueden causar aumento de turbidez, pero no colonias } \\
\text { visibles }\end{array}$ \\
\hline
\end{tabular}

Fuente: Dirección Nacional de Determinantes de la salud e Investigación, (2011)

\section{Floración Cianobacterial}

Las cianobacterias son organismos que están presentes y dispersos en el agua o en el ambiente, además en concentraciones diversas que varían de acuerdo a los factores limitantes de su crecimiento. 
Según el artículo de Cianobacterias como determinantes ambientales (Para \& Personal, 2015)se menciona que se conoce como bloom o floración al crecimiento rápido de la densidad de la población cianobacterial en el agua, alcanzando niveles tales que se puede observar a simple vista, y afecta en mayor medida a ríos, embalses y lagos, constituyéndose en un peligro potencial para la salud, en particular cuando su uso está destinado para recreación, como fuente para riego o para agua potable.

\section{Clasificación de las Cianobacterias}

La mejor forma de clasificarlos y ordenarlos, es mediante la predominancia de las órdenes, posterior a eso siguen los géneros y por último las especies, las cuales son muy variadas con respecto al género, según las investigaciones que se hicieron en todo el mundo, se han determinado unas 2000 especies de cianobacterias, las cuales viven ya sean en ambientes terrestres o acuáticos, dividiéndose en 3 órdenes predominantes que son las chroococcales, los nostocales y las oscillatoriales (Cabrera, 2011).

A continuación se mostrara una clasificación de las cianobacterias más comunes de agua dulces.

Tabla 2

Clasificación de las cianobacterias más comunes de agua dulce.

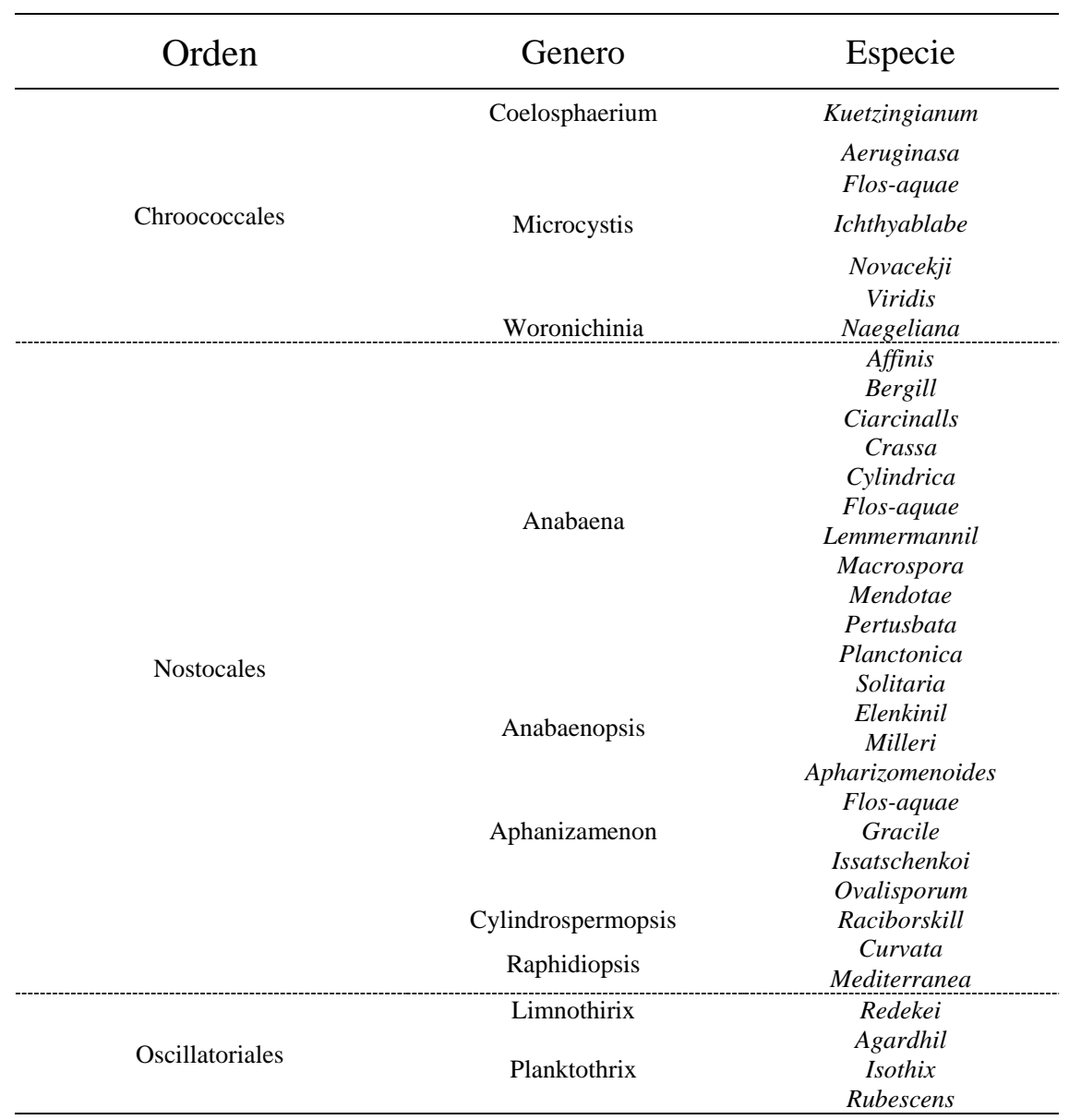

Fuente: Catálogo de cianobacterias planctónicas potencialmente tóxicas de las aguas continentales españolas (2011)

\section{Cianotoxinas}

Un aspecto muy importante sobre las cianobacterias es la presencia de metabolitos secundarios en muchos de los taxones que, en algunas ocasiones, pueden ser tóxicos para los otros organismos de los ecosistemas, incluyendo los humanos. Muchas especies de cianobacterias producen toxinas, las cuales son contenidas en la célula o exudadas al medio, por lo que pueden aparecer disueltas en el agua, constituyéndose en un problema para la salud humana y ambiental (Leda, 2011). 
Estos metabolitos secundarios tóxicos se denominan genéricamente cianotoxinas y se clasifican por los efectos que producen en los animales. Así, las cianotoxinas más nocivas son producías por la Arthrospira fusiformis, Anabaenopsis milleri y Aphanocapsa sp, tres cianobacterias que se reportan en la literatura como altamente tóxicas y cuyas adaptaciones morfológicas, autecología, uso efectivo de nutrientes, espacio y luz, y las consecuencias de su actividad fisiológica en el ecosistema determinaron su prevalencia en la laguna (Lake et al., 2017), por una parte, las hepatotoxinas, que afectan a las células del hígado, representadas fundamentalmente por las microcistinas y las nodularinas (pequeños oligopéptidos de 7 ó 5 aminoácidos) y, por otra, las neurotoxinas, que afectan a la transmisión del impulso nervioso y que son las saxitoxinas y las anatoxinas (pequeños alcaloides). Existen otros grupos de toxinas importantes como son las citotoxinas, fundamentalmente la cilindrospermopsina, y las dermatotoxinas entre las que se encuentra la aplisiatoxina. Existe otra serie de compuestos que tienen toxicidad y que se encuentran en la mayoría de las cianobacterias: las endotoxinas (LPS), que forman parte de la pared de las cianobacterias y que presentan una toxicidad limitada, y el BMAA, que es un aminoácido que parece estar relacionado con enfermedades nerviosas y neurodegenerativas en vertebrados.

Tabla 3 Clasificación de las cianotoxinas según (Tapia, 2015).

\begin{tabular}{|c|c|c|}
\hline Grupo de toxinas & Órgano dañado en mamíferos & $\begin{array}{c}\text { Géneros de cianobacterias productoras de } \\
\text { toxinas }\end{array}$ \\
\hline \multicolumn{3}{|l|}{ Péptidos cíclicos } \\
\hline Microcystinas & Hígado & $\begin{array}{l}\text { Microcystis, Anabaena, Planktthrix } \\
\text { (Oscillatoria), Nostoc, } \\
\text { Hapalosiphon, Anabaenopsis }\end{array}$ \\
\hline $\begin{array}{l}\text { Nodularina } \\
\text { Alcaloides }\end{array}$ & Hígado & Nodularía \\
\hline Anatoxina-a & Sinapsis colinérgicas & $\begin{array}{c}\text { Anabaena, Planktothrix (Oscillatoria), } \\
\text { Apharizomenon }\end{array}$ \\
\hline Anatoxina-a(S) & Sinapsis colinérgicas & Anabaena \\
\hline Aplyslatoxins & Piel & $\begin{array}{l}\text { Lynbya, Schizothrix, Planktothrix } \\
\text { (Oscillatoria) }\end{array}$ \\
\hline Cylindrospermopsinas & Hígado & $\begin{array}{c}\text { Cylindrospermopsis, Aphanizomenon } \\
\text { (Umezakia) }\end{array}$ \\
\hline Lyngbyatoxin-a & Piel, tracto gastrointestinal & Lyngbya \\
\hline Saxitoxinas & $\begin{array}{c}\text { Axones neuronales, inhibe la } \\
\text { conducción } \\
\text { del impulso nervioso }\end{array}$ & $\begin{array}{c}\text { Anabaena, Aphanizomenon, Lyngbya, } \\
\text { Cylindrospermopsis }\end{array}$ \\
\hline $\begin{array}{l}\text { Lipopolisacáridos } \\
\text { (LPS) }\end{array}$ & $\begin{array}{l}\text { Potencial irritante, afecta } \\
\text { cualquier tejido } \\
\text { expuesto }\end{array}$ & Todas \\
\hline
\end{tabular}

Fuente: Antonio Quesada (2011)

\section{Importancia ecológica}

La importancia ecológica de estos organismos radica en la capacidad de generar oxígeno formado durante el proceso fotosintético, esto confirma su importancia como organismos productores fototróficos responsables de generar la atmósfera inicial en el planeta.

Además, generan materia orgánica para otros organismos, son de utilidad económica en suelos donde se cultiva arroz, ya que, al incorporar el nitrógeno atmosférico en compuestos utilizables por estas plantas, se evita la utilización de fertilizantes, se mejora la calidad del suelo y se incrementa el rendimiento agrícola. Algunas cianobacterias establecen relaciones simbióticas con otros organismos tales como, protozoarios, 
hongos (líquenes) y algunas plantas. Cabe resaltar un dato interesante: en los líquenes las cianobacterias carecen de pared celular y funcionan como cloroplastos que producen alimentos para el socio simbiótico.

\section{Eutrofización}

Según Espósito et al., (2016) la descarga de los nutrientes fósforo y nitrógeno contribuyen a la eutrofización, esto es fundamental ya que las cianobacterias tiene relación con la fijación de nitrógeno, para Quirós, Renella, Boveri, Rosso, \& Sosnovsky, (2002) las lagunas con abundante desarrollo del fitoplancton pero escaso desarrollo de la macrofitia coincide con lagunas verdes y "turbias", y que generalmente presentan altas abundancias de peces planctívoros y estas características también coinciden con la laguna estudiada, la aparición y persistencia de las floraciones en cuerpos de agua se consideran un riesgo potencial para la salud de los habitantes del área (Almanza et al., 2016).

\section{Materiales y Métodos}

\subsection{Instrumentos}

El equipo utilizado fue el Oxímetro Hanna (modelo HI9146-04), con el método sonda con electrodos, también se usa el Papel pH, y para capturar muestras se usa mallas estándar de $40 \mathrm{~cm}^{2}$, hilo nailon, para trasladar las muestras se utiliza botellas de $225 \mathrm{ml}$ de vidrio esterilizadas.

\subsection{Diagnóstico de la laguna de la mansión}

Antes de empezar con el muestreo, es necesario tener Información de la laguna la cual se encuentra ubicada a 580 msnm., a una latitud 0299741 S, UTM 8672262.

Gracias a Cruz et al., (2016) se tiene la siguiente data en la Tabla 4.

Tabla 4

Diagnóstico del campo para el tratamiento de agua.

\begin{tabular}{lcc}
\hline Criterios de diseño & Unidad de medida & Cantidad \\
\hline Área total de la laguna & $\mathrm{m}^{2}$ & 3499 \\
Perímetro del lago & $\mathrm{m}$ & 278.86 \\
Volumen de & $\mathrm{m}^{3}$ & 6405.336 \\
almacenamiento & $\mathrm{m}^{3} / \mathrm{sg}$ & 0.12 \\
Caudal de ingreso & semanal & 1 \\
Periodo de ingreso & & \\
\hline
\end{tabular}




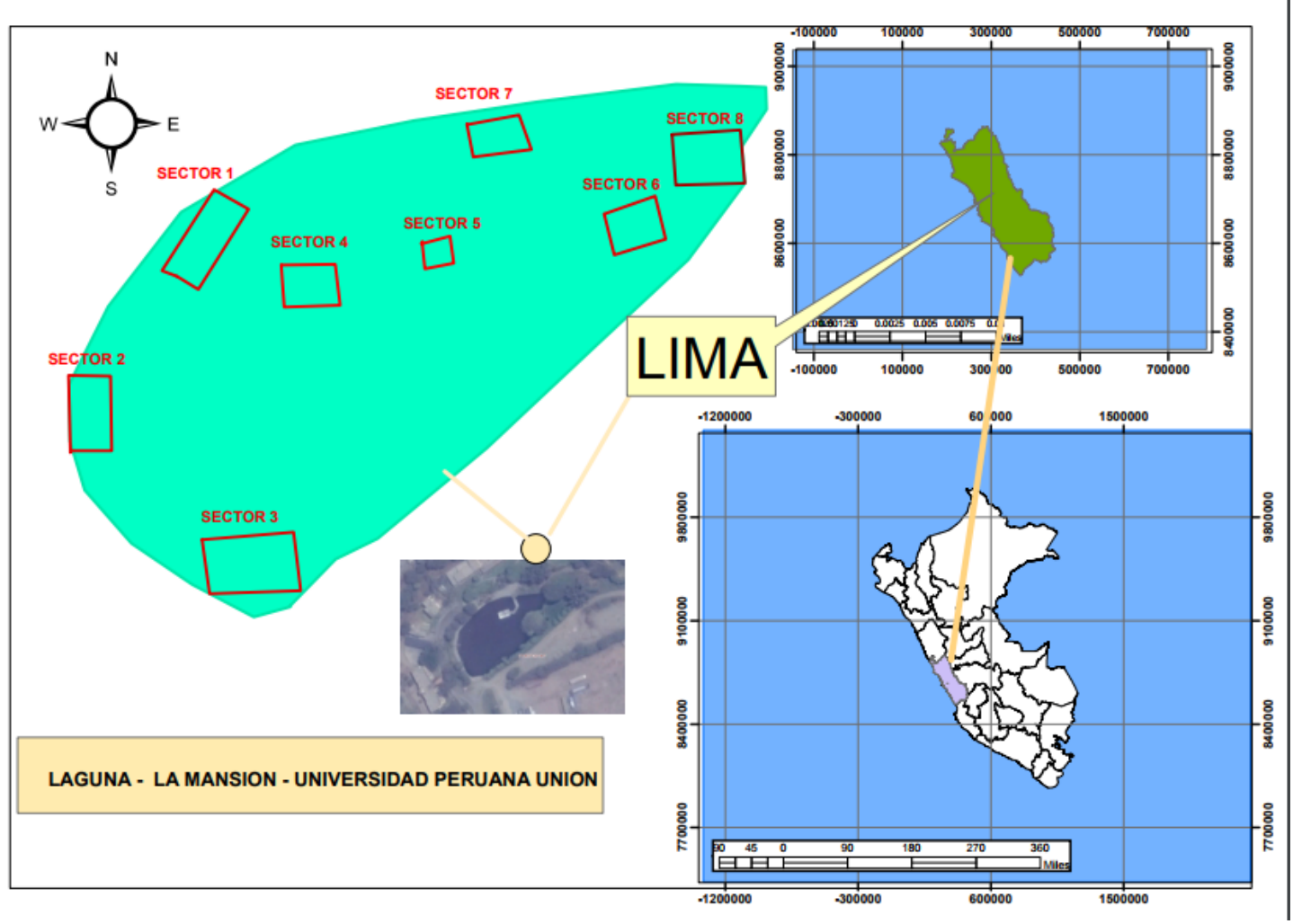

Figura 1 Sectores de colocación de mallas

\subsection{Datos para identificación}

El método empleado para identificar cianobacterias tuvo como base un catálogo con las principales fotos de cianobacterias así se compara, se observa y se registra a que género pertenece Para esto se toma en cuenta los principales géneros de cianobacterias de agua dulce que son: Coelosphaerium, Microcystis, Woronichinia, Anabaena, Anabaenopsis, Aphanizamenon, Cylindrospermopsis, Raphidiopsis, Limnothirix, Planktothrix.

\subsubsection{Colocación de mallas estándares en cada punto especifico}

Para tener un mejor control de la laguna, se muestrea en 8 puntos específicos los cuales están repartidos equilibradamente, estas mallas tienen el objetivo de capturar en su superficie diferentes tipos de protozoos y lo más importante que la cianobacteria pueda crecer en esta malla, la malla estándar es de $20 \mathrm{~cm}$ x $20 \mathrm{~cm}$ de longitud hilo nailon de 2 metros que ira bien asegurado a las mallas. 


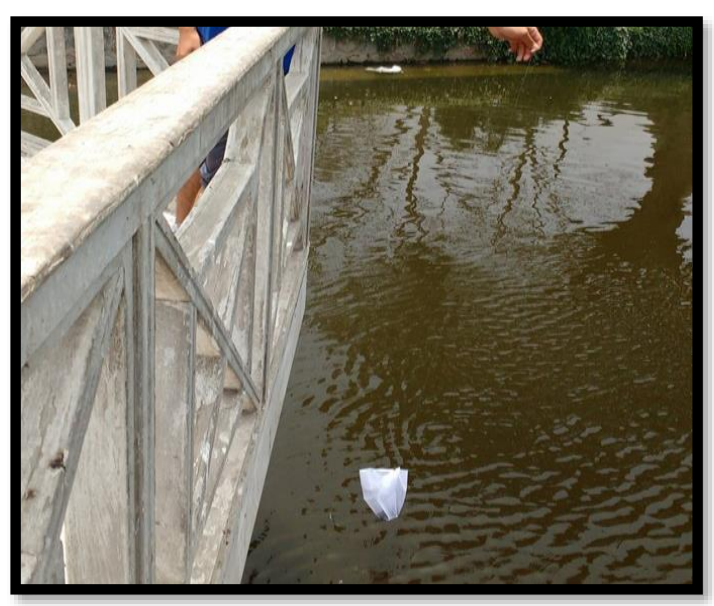

Figura 2 Malla siendo sometida la superficie de la laguna.

\subsubsection{Recolección de muestras}

Para este paso, el proceso es un poco más fácil, pero se debe tomar en cuenta medidas de bioseguridad, siempre estar bien protegidos con guantes y mascarilla, pues se sabe que algunas cianotoxinas pueden liberar esporas tóxicas, solo tenemos que sacar la malla de la laguna, luego en una botella de vidrio de $225 \mathrm{ml}$ previamente esterilizada y llenada con agua de ese mismo punto, lo que hacemos es enjuagar la malla con todo el material orgánico que se encuentre dentro de ella, y así para cada punto hasta obtener las 8 muestras que deseamos.

\subsubsection{Toma de parámetros, oxígeno y $\mathrm{pH}$}

Con el uso de un Oxímetro y el papel pH se obtienen los datos de cada uno de los puntos, ya que son de mucha importancia para saber qué condiciones favorecen al desarrollo de las cianobacterias.

Por tal motivo se utiliza la siguiente tabla, con el objetivo determinar si el Oxígeno Disuelto está beneficiando o perjudicando al ecosistema acuático en estudio.

Tabla 5 Rangos de concentración de Oxígeno disuelto y consecuencia ecosistémitas frecuentes.

\begin{tabular}{lcc} 
[OD] mg/L & Condición & Consecuencias \\
\hline 0 & Anoxia & Muerte masiva de organismos aerobios \\
$0-5$ & Hipoxia & $\begin{array}{c}\text { Desaparición de organismos y especies sensibles } \\
\text { [OD] adecuadas para la vida de la gran mayoría de } \\
\text { Especies de peces y otros organismos acuáticos }\end{array}$ \\
$5-8$ & $\begin{array}{c}\text { Aceptable } \\
\text { Buena }\end{array}$ & $\begin{array}{c}\text { Sistemas en plena producción fotosintética. } \\
>12\end{array}$ \\
\hline
\end{tabular}

Fuente: Guillermo Goyenola; 2007 


\subsubsection{Observación microscópica de las muestras}

Finalmente se filtra el agua de la laguna en un matraz, con la ayuda de un papel filtro, o también se puede agitar muy bien la botella llena de agua, y con la ayuda de una pipeta de platico, se saca una pequeña muestra, esta muestra la llevamos a una lámina portaobjeto que posteriormente será estudiada en un microscopio.

\subsubsection{Tinción Gram para diferenciar cianobacterias de algas}

Las cianobacterias son bacterias Gram Negativas, por lo que si deseamos diferenciarlas de las algas, necesitaremos hacer tinción Gram que consiste en teñir las muestras con diferentes colorante que permiten teñir su pared celular y diferenciarla.

\subsubsection{Tinción con azul de metileno para una mejor vista a su estructura}

Para tener una mayor seguridad de la presencia de la cianobacteria, se agregar Azul de Metileno a la muestra antes de observarlo en el microscopio, esta tinción nos permite ver con una mayor nitidez la estructura de las cianobacterias y así diferenciar mejor su género.

\subsection{Análisis estadístico}

Es necesario que parte de los resultados más resaltantes sean analizados, para buscar relación entre las variables trabajadas.

En el desarrollo de la ciencia en general y en especial en el de las ciencias biológicas, el conocimiento de la metodología estadística es una arma imprescindible para la obtención, análisis e interpretación de todos los datos que proceden de las observaciones sistemáticas o de experimentaciones proyectadas específicamente para conocer los efectos de uno o varios factores que intervienen en los fenómenos bajo estudio. (Castillo \& Cortez, 2007)

Este trabajo de investigación contiene variables cualitativas, es por ello que, se utiliza la técnica de la prueba de Chi-cuadrado.

\section{Resultados y Discusión}

\subsection{Captura de microorganismos y partículas suspendidas en el agua}

A las 24 horas las mallas presentan ligeras manchas a visión macroscópica lo que se ha capturado son residuos de plantas maderitas pajitas y un poquito de algas. 


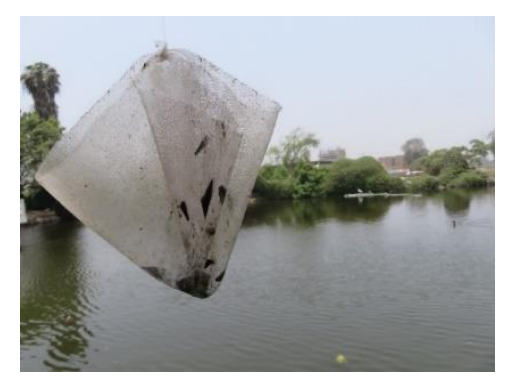

Figura 3 Observación de mallas a las 24 horas

A las 72 horas en ciertos puntos especialmente a las orillas como es el punto 7 seis y ocho se capturaron más partículas suspendidas en el agua y microorganismos.

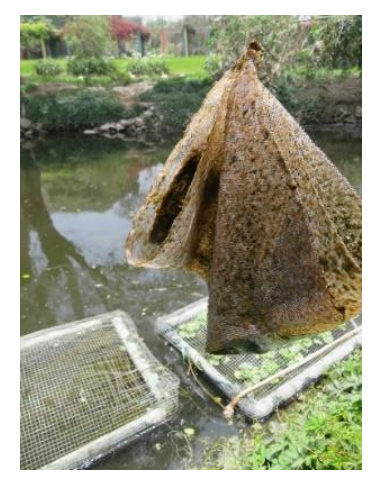

Figura 4 Acumulación de microorganismos y partículas suspendidas en el agua en la malla del punto 6

Después de tres semanas todos los puntos presentan coloración verdosa y como posteriormente lo comprobamos en laboratorio tienen cianobacterias. La mallas se ven como las del punto 8 que se aprecia en las figura 5.

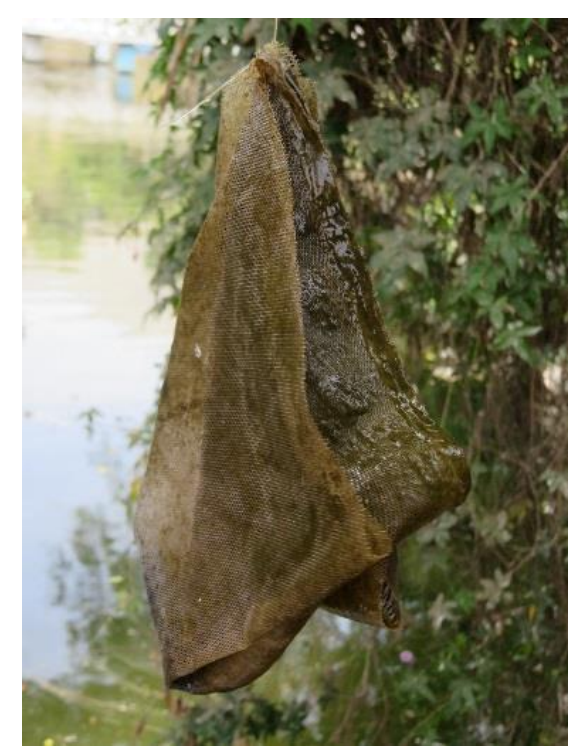

Figura 5 Malla del punto 8 después de tres semanas

Con la captura de estas mallas se aseguró tener más muestra para observar, y que no solo se tomó un punto reducido si no que con este método se captura todo lo que pueda estar por lo menos en un metro cuadrado de acuerdo al movimiento que tenga la malla. Así los resultados para muestra se plasman en la figura 6. Botella con contenido lista para llevar a ser observada. 


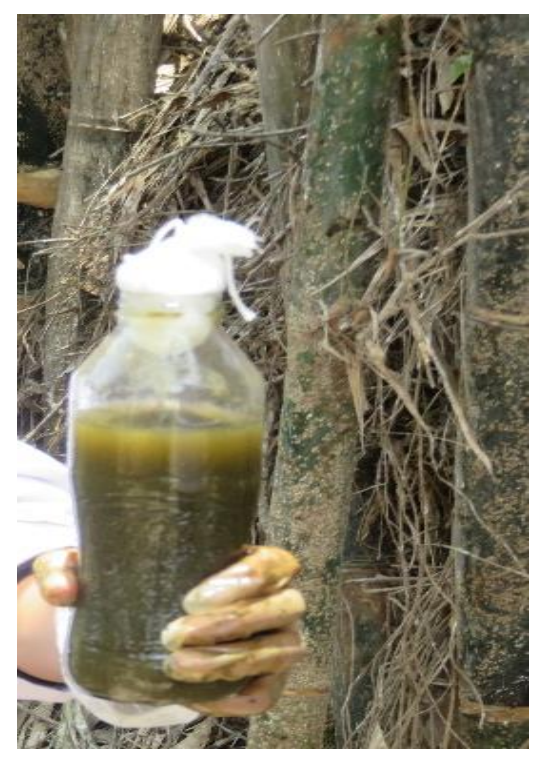

Figura 6 Botellas de $225 \mathrm{ml}$ con muestra representativa para ser observada al microscopio.

\subsection{Cianobacterias identificadas en la laguna "La Mansión"}

En la laguna de la mansión se encontraron diferentes géneros de cianobacterias en diferentes puntos

Tabla 6

Cianobacterias encontradas en los puntos ubicados en la laguna

Géneros de cianobacterias

$$
\text { Aphanizamenon Anabaena Limnothirix Microcystis Planktothrix Raphidiopsis }
$$

\begin{tabular}{lcccccc}
\hline Punto 1 & NO & SI & NO & SI & NO & NO \\
Punto 2 & NO & NO & SI & NO & NO & NO \\
Punto 3 & SI & NO & NO & NO & NO & NO \\
Punto 4 & NO & NO & NO & NO & NO & SI \\
Punto 5 & NO & NO & NO & NO & SI & NO \\
Punto 6 & NO & SI & NO & NO & NO & NO \\
Punto 7 & NO & SI & NO & NO & NO & NO \\
Punto 8 & NO & SI & NO & NO & NO & NO \\
\hline
\end{tabular}


La principal cianobacteria encontrada fue la especie de la Anabaena que se encontró en los puntos más verdosos, además de cianobacterias abundan protozoos, como es el caso de Stentor identificado en el punto 7 y 6.

\subsection{Resultado de la tinción Gram para diferenciar algas de cianobacterias}

En el punto uno se encontró una zona muy verdosa y al tomar las muestras se presenta la pregunta si es cianobacteria o alga, es por ello que a través de la técnica de tinción Gram determinamos que las muestras contenían cianobacterias ya que se tornaron rosas confirmando que son Gram negativas, también se encontraron algas, pero se sabe cianobacterias conviven con estas.

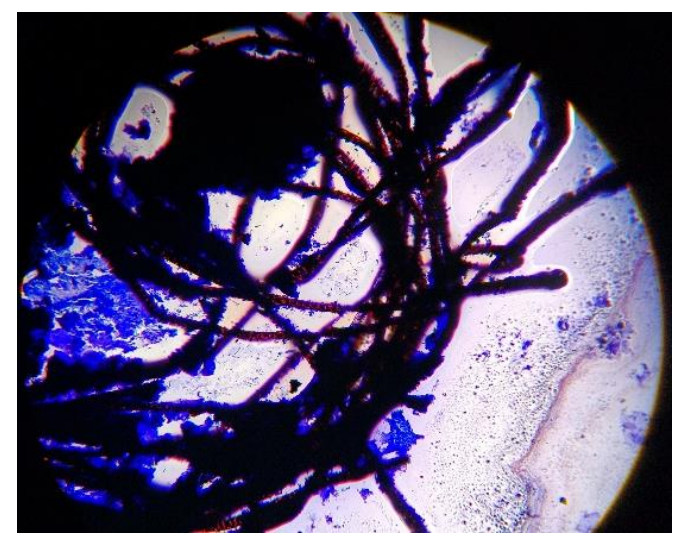

Figura 7 Muestra del punto 1 sometida a tinción Gram

\subsection{Medición de parámetros de oxígeno, pH y temperatura}

\section{Tabla 7}

Tabla con los datos de pH y OD de la alguna de la mansión.

\begin{tabular}{lccc}
\hline $\begin{array}{c}\text { Sectores de la } \\
\text { laguna }\end{array}$ & Ph de la laguna & OD de la laguna & Temperatura \\
\hline Sector 1 & 6,5 & 15,3 & $27.3^{\circ} \mathrm{C}$ \\
Sector 2 & 7,0 & 13,0 & $27^{\circ} \mathrm{C}$ \\
Sector 3 & 6,5 & 13,5 & $26.4{ }^{\circ} \mathrm{C}$ \\
Sector 4 & 6,5 & 16,5 & $25.5^{\circ} \mathrm{C}$ \\
Sector 5 & 6,5 & 15,2 & $24.8^{\circ} \mathrm{C}$ \\
Sector 6 & 7,0 & 9,4 & $24.4{ }^{\circ} \mathrm{C}$ \\
Sector 7 & 7,5 & 11,8 & $25.8^{\circ} \mathrm{C}$ \\
Sector 8 & 7,5 & 10,1 & $26.3{ }^{\circ} \mathrm{C}$ \\
\hline
\end{tabular}

Nota: $O D=$ Oxígeno Disuelto 
Estos datos fueron tomados entre las 9 y 10 de la mañana ya entrando a estación de verano (Lima-Perú) por lo que el sol intenso sube la temperatura del agua. Además, beneficia a las cianobacteria para que esta pueda realizar fotosíntesis.

\subsection{Resultados estadísticos}

\subsubsection{Análisis entre Cianobacterias de agua dulce y Orden a la que pertenece}

\section{Tabla 8}

Tabla de contingencia entre la variable de Género de Cianobacteria y Efecto de la Cianobacteria en agua dulce.

Especie de Cianobacteria*Efecto de las cianotoxinas en el agua tabulación cruzada

\begin{tabular}{lcccc}
\hline & $\begin{array}{c}\text { Efecto de las cianotoxinas en } \\
\text { el agua }\end{array}$ & Toxicidad & Olor & Total \\
Especie de Cianobacteria & 6 & 0 & 0 & 6 \\
Anabaena & 0 & 1 & 0 & 1 \\
Aphanizamenon & 0 & 0 & 1 & 1 \\
limnothirix & 0 & 1 & 0 & 1 \\
microcystis & 0 & 1 & 0 & 1 \\
Planktothrix & 0 & 1 & 0 & 1 \\
raphidiopsis & 0 & 1 & 0 & 1 \\
Total & 6 & 5 & 1 & 12 \\
\hline
\end{tabular}

Tabla 9

Resultado de hacer la prueba de chi cuadrado, siendo el valor del Sig. Menor a 0.5.

\section{Pruebas de chi-cuadrado}

Sig.

asintótica

\begin{tabular}{lccc} 
& Valor & gl & (bilateral) \\
Chi-cuadrado de Pearson & $24,000^{\mathrm{a}}$ & 12 & .020 \\
Razón de verosimilitudes & 22.042 & 12 & .037 \\
\hline
\end{tabular}

Al tener el valor del Sig. (0,020), el cual es menor al nivel de significancia de 0,5 , se ha compruea la dependencia de la variable de Efectos de cianotoxinas a la variable de Género de cianotoxinas.

Entonces se concluye estadísticamente que el género de cianobacterias Aphanizamenon, es la que causa el mal olor en la laguna de la "Mansión", y los géneros Anabaena, Limnothirix microcystis, Planktothrix, raphidiopsis, son las que causan la toxicidad en la laguna. 


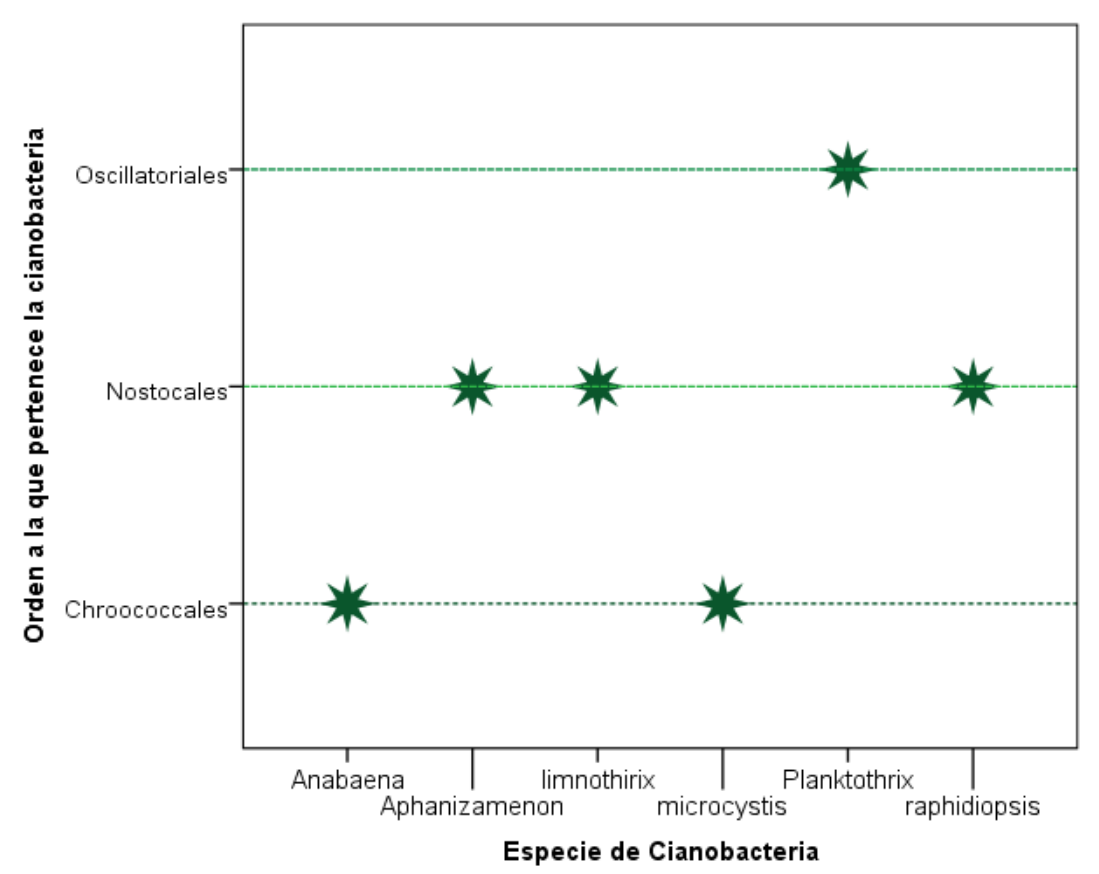

Figura 8 Gráfica donde se muestra la relación que hay dos variables cualitativas.

\subsubsection{Análisis entre pH y OD de la laguna}

Tabla 10

Datos estadísticos sobre la tendencia de correlación que siguen las variables.

\begin{tabular}{|c|c|c|c|}
\hline \multicolumn{4}{|c|}{ Correlaciones } \\
\hline & & $\begin{array}{c}\text { pH de la laguna } \\
\text { Mansión }\end{array}$ & $\begin{array}{c}\text { Oxígeno disuelto } \\
\text { de la laguna }\end{array}$ \\
\hline $\mathrm{pH}$ de la laguna & $\begin{array}{l}\text { Correlación de } \\
\text { Pearson }\end{array}$ & 1 &,$- 785^{*}$ \\
\hline & Sig. (bilateral) & & .021 \\
\hline $\begin{array}{l}\text { Oxígeno } \\
\text { disuelto de la }\end{array}$ & $\begin{array}{l}\text { Correlación de } \\
\text { Pearson }\end{array}$ &,$- 785^{*}$ & 1 \\
\hline laguna & Sig. (bilateral) & .021 & \\
\hline
\end{tabular}

Al tener el valor de correlación de Pearson negativa como $-7,85$, se determina que al $95 \%$ de nivel de confianza, se acepta los datos pertenecientes a una correlación negativa fuerte, además el tipo de relación que tienen ambas variables son inversamente proporcionalmente, esto se puede ver claramente en el siguiente grafico de dispersión de puntos lineal negativo. 


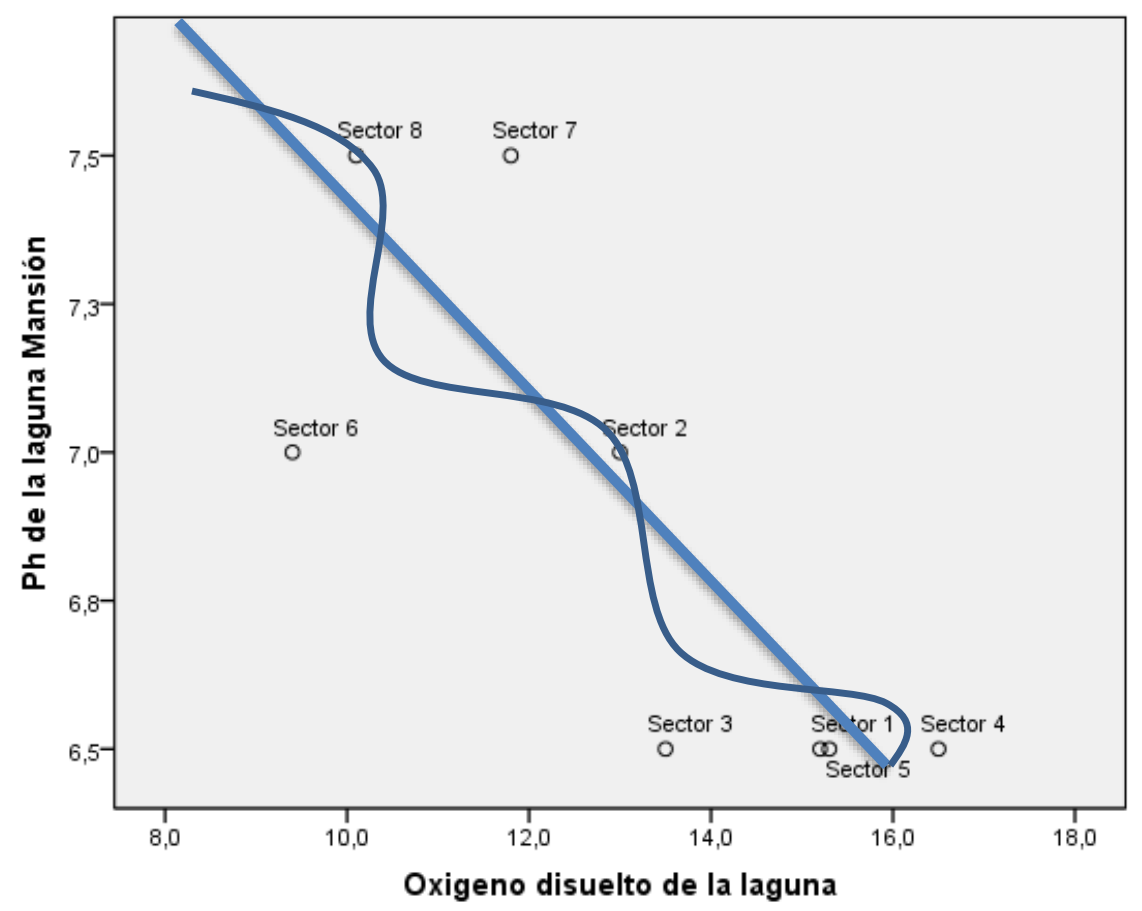

Figura 9 La siguiente grafica nos muestra que la dispersión de puntos sigue una correlación lineal negativa fuerte.

\subsubsection{Discusión}

Los 6 géneros de Cianobacterias encontrados en la alguna de "La Mansión" son los más frecuentes en ecosistemas de agua dulce, se confirma que estas cianobacterias son las responsables de fijar nitrógeno y aportar oxígeno al ecosistema local, es por ello que hay una pequeña diversidad acuática y fauna, pues estas cianobacterias son la base primordial de la cadena trófica, debido a que son las que inician el proceso trófico (J, 2001).

Además el género Anabaena de las cianobacterias son las que más abundan en esta laguna, pues estas son capaces de soportar temperaturas extremas de hasta $70^{\circ} \mathrm{C}$ (Susana, 1999), por lo que si no hay un buen manejo o control de las aguas de esta laguna, es seguro predecir la eutrofización de esta laguna, ocasionando la muerte de especies acuáticos que actualmente interactúan con esta cianobacteria, tales como los peces tilapia, una tortuga, y una gran variedad de protozoos.

El Stentor es un ciliado que abunda en la laguna de la Mansión, pues durante los análisis para identificar las especies de cianobacterias, este protozoo aparece en cantidades significativas, siempre cerca de las 6 especies de cianobacterias identificadas, según (Guillen, 2010), la relación que hay entre el Stentor y las cianobacterias es una relación inter especifica comensalista, pues mientras las cianobacterias junto con otras bacterias descomponen la materia orgánica, los stentors son los más beneficiados porque ellos son los que se alimentan de la descomposición orgánica, los cuales les ofrece proteínas y azucares.

Una de las técnicas que se puede usar para evitar el afloramiento de las cianobacterias, es de manera biológica, mediante el uso de bacterias que lisan las células de las cianobacterias, estas bacterias son denominadas actinobacterias, y el género Actinomicetos Streptomyces es una actinobacteria que puede actuar produciendo factores antibióticos y agentes líticos, para un control biológico en la inhibición de las cianobacterias, mediante el uso de pajas de cebada donde hay una amplia gama de microrganismos que producen sustancias antimicrobianas, las cuales producen una muerte del 50\% de las cianobacterias que pertenecen al género de 
Anabaena, Microsystis y Oscillatorias (Cobo, 2015), permitiendo un mejor manejo natural frente a la problemática que produce el excesivo crecimiento de las cianobacterias.

Las cianobacterias tienen la capacidad de adaptarse diferentes ambientes, según las condiciones que se le presenten, es por ello que (Pompeo, Moschini, \& Yuri, 2015) menciona, que en la represa Billings y en el rio Taquacetuba, Sao Paulo-Brasil, las cianobacterias son consideradas discriminadoras de la calidad del agua, debido a que cianobacterias como Raciborskii y Pagardhii pueden soportar poca luz solar, y alimentarse de amonio para subsistir y seguir reproduciéndose en el agua, además considera a Microcystis y a la Planktothrix, como cianobacterias discriminadoras de la calidad de agua, siendo estas identificadas en la laguna de la Mansión.

La presencia de los peces Tilapia en la laguna de la Mansión, nos muestran que ellos son parte también de la cadena trófica que es iniciada por las cianobacterias, según (Quiros, Rennella, Boveri, Rosso, \& Sosnovsky, 2002), las lagunas verdes y turbias que presentan floraciones de algas y cianobacterias tienen presencia de peces planctívoros como es el caso de la Tilapia.

\section{Conclusiones}

Finalmente se identificó seis tipos de cianobacterias presentes en la laguna de la Mansión, las cuales causan un efecto en el ecosistema acuático, como el mal olor en la laguna causado por la cianobacteria de genero Aphanizamenon. Los géneros hallados en más de 50\% producen cianotoxinas, lo que indica una producción excesiva en el ecosistema acuático. El oxígeno en la laguna indica saturación ligera, en algunos puntos más que en otros, las condiciones que está tomando la laguna preparan un ambiente apto para la sobrepoblación de cianobacterias lo cual generaría una eutrofización que sería causante de la muerte acuática de los seres vivos macroscópicos.

\section{Recomendaciones}

Se recomienda realizar monitoreos constantes para disminuir la posibilidad de eutrofización del agua, asimismo se realizaría una cuantificación de la diversidad de las cianobacterias, mediante el índice de Shannon y Simpson.

\section{Referencias}

Almanza, V., Parra, O., De Carlos Bicudo, C. E., Baeza, C., Beltran, J., Figueroa, R., \& Urrutia, R. (2016). Occurrence of toxic blooms of Microcystis aeruginosa in a central Chilean ( $36^{\circ}$ Lat. S) urban lake. Revista Chilena de Historia Natural, 89, 1-12. https://doi.org/10.1186/s40693-016-0057-7

Alonso, C. D. E. H., Vill, L., Fuente, D. E. L. A., Mart, D., Pozo, D. E. L., Bueno, L. C., ... Avil, J. (2008). Problemas de las cianobacterias en aguas de recreo y aguas de consumo, 63-69.

Cabrera, D. (2011). Evaluación de la relación entre las densidades poblacionales de cianobacterias (mycrocistis sp, anabaena sp, oscillatoria sp.) y las concentraciones de los parámetros fisicoquímicos: nitratos y fosfatos en el lago de amatitlán, guatemala durante los meses. universidad de san carlos de guatemala. Universidad de San Carlos de Guatemala. Retrieved from http://biblioteca.usac.edu.gt/tesis/06/06_3113.pdf

Castillo, J., \& Cortez, K. (2007). Estadística \&amp; investigación Papel de la estadística en la investigación 
científica (Role of statistics in scientific research). InnOvaciOnes de NegOciOs, 4(1), 107-145. https://doi.org/ISSN 1665-9627

Cobo, F. (2015). Métodos de control de las floraciones de cianobacterias en aguas continentales . AIL, (August). Retrieved from

https://www.researchgate.net/profile/Fernando_Cobo/publication/278962250_Metodos_de_control_de _las_floraciones_de_cianobacterias_en_aguas_continentales/links/55c881b208aeca747d66c6c5/Metod os-de-control-de-las-floraciones-de-cianobacterias-en-aguas-continentales.pdf

Cruz, M., Carbo, N., Gonzales, J. L. L., Tito, G. M., Depaz, K., Torres, S., ... Quispe, W. (2016). Tratamiento De Las Aguas De La Laguna “ Mansión ” Mediante La Especie Eichhorniacrassipes , Para El Riego De Áreas Verdes En La Universidad Peruana Unión. Journal of Agriculture and Veterinary Science, 9(8), 53-65. https://doi.org/10.9790/2380-0908025365

Determinantes, C., \& La, A. D. E. (2011). Como determinantes ambientales de la salud edición 2011 serie: temas de salud ambiental $n^{\circ} 5$ departamento de Salud Ambiental.

Espósito, M. E., Blanco, M. C., Sequeira, M. E., Paoloni, J. D., Fernández, S. N., Amiotti, N. M., \& Díaz, S. L. (2016). Natural pollution (As, F) and eutrofication (N, P) in the basin of El Divisorio stream, Argentina. Phyton, 85(2016), 51-62.

González-piana, M. (2012). Efecto de la intensidad lumínica y la temperatura sobre el crecimiento y la morfología de dos cianobacterias filamentosas formadoras de floraciones. Facultad de Ciencias UDELAR. Retrieved from http://www.bib.fcien.edu.uy/files/etd/resumen/uy24-16101R.pdf

Guillen, A. (2010). La biodiversidad oculta. Paginas de Informacion Ambiental, 24-29. Retrieved from Dialnet-LaBiodiversidadOculta-3292247 (1).pdf

Hidrol, P. (n.d.). Cianobacterias Planctónicas del Uruguay.

J, R. (2001). Detección de cianobacterias y sus toxinas . Una revisión. Toxicologia, 1, 65-71. Retrieved from http://www.accede.org/papers/cianobacterias.pdf

Lake, S., Morales, E. A., Rivera, S. F., Vildozo, L. H., Pol, A., Criptogámico, H., .. Box, P. O. (2017). Floración algal nociva ( FAN ) producida por cianobacterias en la laguna Alalay , Cochabamba ,. Universidad Catolica Boliviana San Pablo, 8, 50-75. Retrieved from https://www.researchgate.net/publication/318681746_Floracion_algal_nociva_FAN_producida_por_ci anobacterias_en_la_laguna_Alalay_Cochabamba_Bolivia_Harmful_algal_bloom_HAB_produced_by _cyanobacteria_in_Alalay_Shallow_Lake_Cochabamba_Bolivia

Leda, G. (2011). Como determinantes ambientales de la salud edición 2011 serie: temas de salud ambiental $n^{\circ} 5$ departamento de Salud Ambiental (Ministerio). Buenos Aires. Retrieved from http://www.msal.gob.ar/images/stories/bes/graficos/0000000334cnt-05-Cianobacterias.pdf

Para, C., \& Personal, E. L. (2015). Cianobacterias como determinantes ambientales de la salud. Ministerio de Salud, primeroa.

Pompeo, M., Moschini, C., \& Yuri, P. (2015). A comunidade fitoplanctônica como discriminador da qualidade da água na represa Billings (são paulo, sp). Ecologia de Reservatórios E Interfaces, São Paulo : Instituto de Biociências Da Universidade de São Paulo, 49-58. Retrieved from http://ecologia.ib.usp.br/reservatorios/PDF/Cap._3_Comunidade_fitoplanctonica.pdf

Quirós, R., Renella, A. M., Boveri, M. B., Rosso, J., \& Sosnovsky, A. (2002). Factores que afectan la estructura y el funcionamiento de las lagunas pampeanas. Ecología Austral, 12, 175-185.

Quiros, R., Rennella, A., Boveri, M., Rosso, J., \& Sosnovsky, A. (2002). Factores que afectan la estructura y el funcionamiento de las lagunas pampeanas. Azociacion Argentina de Ecologia, 175-185. Retrieved from http://www.scielo.org.ar/pdf/ecoaus/v12n2/v12n2a11.pdf 
Ramirez, R., Rojo, M., \& Espejel, F. (2007). Remoción de cianobacterias mediante el proceso de flotación por aireación. Intituto de Ingenieria UNAM, 2(1), 550-555. Retrieved from http://web.uaemex.mx/Red_Ambientales/docs/congresos/CHIHUAHUA/docs/101-120.pdf

Tapia, C. (2015). Caracterización de las toxinas obtenidas de las cianobacterias presentes en los estanques de producción de peces de la estación acuícola de repelón, y el embalse El Guájaro, Atlántico. Universidad de La Costa 1970, (137). Retrieved from http://www.aunap.gov.co/wpcontent/uploads/2016/10/16-INFORME-FINAL-CIANOBACTERIAS.pdf 